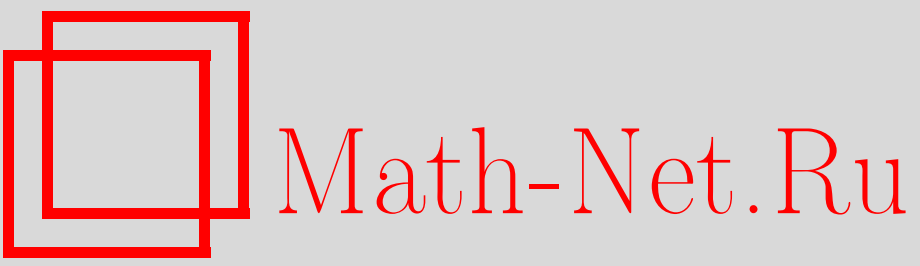

Kh. Es-Sebaiy, C. A. Tudor, Noncentral limit theorem for the cubic variation of a class of self-similar stochastic processes, Теория вероятн. и ее примен., 2010, том 55, выпуск 3, 507-529

DOI: https://doi.org/10.4213/tvp4239

Использование Общероссийского математического портала Math-Net.Ru подразумевает, что вы прочитали и согласны с пользовательским соглашением

http://www. mathnet.ru/rus/agreement

Параметры загрузки:

IP : 54.174 .149 .18

26 апреля 2023 г., 14:09:16 


\title{
NONCENTRAL LIMIT THEOREM FOR THE CUBIC VARIATION OF A CLASS OF SELF-SIMILAR STOCHASTIC PROCESSES
}

\begin{abstract}
Используя кратные стохастические интегралы Винера-Ито, мы изучаем вариацию третьего порядка одного класса самоподобных (автомодельных) процессов со стационарными приращениями (процесс Розенблатта с коэффициентом самоподобия $H \in(1 / 2,1))$. Исследование мотивировано статистическими целями. Доказывается, что для ренормированной вариации третьего порядка справедлива нецентральная предельная теорема и предел (в смысле $\left.L^{2}(\Omega)\right)$ снова является процессом Розенблатта.
\end{abstract}

Ключевые слова и фразы: кратные стохастические интегралы, самоподобные (автомодельные) процессы, процесс Розенблатта, фрактальное броуновское движение, нецентральная предельная теорема, исчисление Маллявена.

1. Introduction. The self-similarity property for a stochastic process means that scaling of time is equivalent to an appropriate scaling of space. That is, a process $\left(Y_{t}\right)_{t \geqslant 0}$ is self-similar of order $H>0$ if for all $c>0$ the processes $\left(Y_{c t}\right)_{t \geqslant 0}$ and $\left(c^{H} Y_{t}\right)_{t \geqslant 0}$ have the same finite dimensional distributions. The self-similar processes are of interest for various applications, such as economics, internet traffic or hydrology. The fractional Brownian motion is the usual candidate to model phenomena in which the self-similarity property can be observed from the empirical data. Recall that the fractional Brownian motion is a centered Gaussian process with covariance function $R^{H}(t, s)=\left(t^{2 H}+s^{2 H}-|t-s|^{2 H}\right) / 2$. The parameter $H \in(0,1)$ characterizes almost all important properties of the process. The fractional Brownian motion can also be defined as the only Gaussian process which is self-similar with stationary increments. In some models the Gaussianity assumption

*SAMOS/MATISSE, Centre d'Economie de la Sorbonne, Université de PanthéonSorbonne Paris 1, 90, rue de Tolbiac, 75634 Paris Cedex 13, France; e-mail: Khalifa.EsSebaiy@malix.univ-paris1.fr

${ }^{* *}$ SAMOS/MATISSE, Centre d'Economie de la Sorbonne, Université de PanthéonSorbonne Paris 1, 90, rue de Tolbiac, 75634 Paris Cedex 13, France; Laboratoire Paul Painlevé, U.F. R. de Mathématiques Université de Lille 1, Cité Scientifique, 59655 Villeneuve d'Ascq Cedex, France; e-mail: tudor@math.univ-lille1.fr 
could be not plausible and in this case one needs to use a different selfsimilar process with stationary increments to model the phenomena. Natural candidates are the Hermite processes: these stochastic processes appear as limits in the so-called noncentral limit theorem (see [3], [7], [18], [10]). In contrast with the classical central limit theorem, the noncentral limit theorem deals with sequences of dependent random variable whose renormalized sum converges in some situations to a non-Gaussian distribution. For a complete exposition of limit theorems in probability theory, we refer to [9] or [16]. Except the Gaussian character, these Hermite processes have the same property as the fractional Brownian motion with Hurst parameter $H>1 / 2$ : self-similarity, stationarity of increments, Hölder continuous path, long-range dependence. While the fractional Brownian motion can be expressed as a Wiener integral with respect to the standard Wiener process, the Hermite process of order $q \geqslant 2$ is a $q$ iterated integral of a deterministic function with $q$ variables with respect to the Brownian motion. The Rosenblatt process is obtained in the particular case $q=2$. It will be properly defined in Section 2. This processes have been recently studied by several authors (see [2], [4], [11]-[13], [19], [20]).

The Hurst parameter $H$ characterizes all the important properties of a Hermite process, as seen above. Therefore, estimating $H$ properly is of the utmost importance. Several statistics have been introduced to this end, such as wavelets, $k$-variations, variograms, maximum likelihood estimators, or spectral methods. Information on these various approaches can be found in $[1]$.

One of the most popular methods to estimate the self-similarity order for stochastic processes is based on the study of their variations. The $p$-variation of a process $\left(X_{t}\right)_{t \in[0,1]}$ is defined as the limit of the sequence (sometimes the absolute value of the increment is used in the definition)

$$
V^{p, N}(X)=\frac{1}{N}\left[\sum_{i=0}^{N-1} \frac{\left(X_{(i+1) / N}-X_{i / N}\right)^{p}}{\mathbf{E}\left(X_{(i+1) / N}-X_{i / N}\right)^{p}}-1\right]
$$

There exists a direct connection between the behavior of the variations and the convergence of an estimator for the self-similarity order based on these variations (see [6], [20]); basically if the renormalized variation satisfies a central limit theorem then the estimator satisfies a central limit theorem and this fact is very useful for statistical aspects.

In a recent paper [20] the quadratic variation of the Rosenblatt process $\left(Z_{t}^{(H)}\right)_{t \in[0,1]}$ with self-similarity order $H \in(1 / 2,1)$ has been studied. The following facts happen: the normalized sequence $N^{1-H} V^{2, N}\left(Z^{(H)}\right)$ satisfies a noncentral limit theorem, it converges in $L^{2}$ to the Rosenblatt random variable $Z_{1}^{(H)}$. From this, we can construct an estimator for $H$ whose behavior is still nonnormal. This situation is somehow not good for statistical 
applications because one always prefers the estimators which are asymptotically normal. To have normal estimators we need to define some adjusted variations (as in $[20]$ ).

In the fractional Brownian motion case the well-known nonnormality of the quadratic variation when $H \in(3 / 4,1)$ can be avoided by using «longer filters» (that means replacing the increments $X_{(i+1) / N}-X_{i / N}$ by $X_{(i+1) / N}-$ $2 X_{i / N}+X_{(i-1) / N}$ ) or higher order variations (choosing a bigger $p$ ). In this work we will consider the second choice (the first choice has been treated in [5]): we replace the quadratic variation by the cubic variation for the Rosenblatt processes to see what happens and if it is possible to find a Gaussian distribution as law of the renormalized cubic variation. In the fractional Brownian motion case, this has no sense because the third moment of a centered Gaussian random variable is zero. We use the Wiener chaos expansion for the statistics $V^{3, N}\left(Z^{(H)}\right)$ and we will decompose it in several terms in the Wiener chaoses 2,4 , and 6 . As in other cases [20], [4] the second chaos term is dominant and it has to be renormalized by $N^{1-H}$ to have a nontrivial limit. We note that the rate of convergence $N^{1-H}$ is the same as for quadratic variation, so there is no gain for the speed and moreover the limit is again, modulo a constant, a Rosenblatt random variable with index $H$ (only the constant is changing). This property has been called in [4] the reproduction property of the Rosenblatt process because its variation generates again Rosenblatt random variable as limit. We conjecture that the same property holds true for the $p$-variations.

The organization of our paper is as follows. Section 2 contains the presentation of the basic tools that we will need throughout the paper: multiple Wiener-Itô integrals and their basic properties, the definition of the Rosenblatt process and its characteristics. In Section 3 we estimate the mean square of the cubic variation of the Rosenblatt process and we give its normalization and finally in Section 4 we prove a noncentral limit theorem for the renormalized cubic variation.

\section{Preliminaries.}

2.1. Multiple stochastic integrals. In this subsection we describe the basic elements of calculus on Wiener chaos. Let $\left(W_{t}\right)_{t \in[0,1]}$ be a classical Wiener process on a standard Wiener space $(\Omega, \mathscr{F}, \mathbf{P})$. If $f \in L^{2}\left([0,1]^{n}\right)$ with $n \geqslant 1$ integer, we introduce the multiple Wiener-Itô integral of $f$ with respect to $W$. We refer to [14] for a detailed exposition of the construction and the properties of multiple Wiener-Itô integrals.

Let $f \in \mathscr{S}_{m}$ be an elementary function with $m$ variables that can be written as

$$
f=\sum_{i_{1}, \ldots, i_{m}} c_{i_{1}, \ldots, i_{m}} 1_{A_{i_{1}} \times \cdots \times A_{i_{m}}},
$$

where the coefficients satisfy $c_{i_{1}, \ldots, i_{m}}=0$ if two indices $i_{k}$ and $i_{l}$ are equal 
and the sets $A_{i} \in \mathscr{B}([0,1])$ are disjoints. For such a step function $f$ we define

$$
I_{m}(f)=\sum_{i_{1}, \ldots, i_{m}} c_{i_{1}, \ldots, i_{m}} W\left(A_{i_{1}}\right) \cdots W\left(A_{i_{m}}\right)
$$

where we put $W([a, b])=W_{b}-W_{a}$. It can be seen that the application $I_{n}$ from $\mathscr{S}$ to $L^{2}(\Omega)$ is an isometry on $\mathscr{S}$, i.e.,

$$
\mathbf{E}\left[I_{n}(f) I_{m}(g)\right]=n !\langle f, g\rangle_{L^{2}\left([0,1]^{n}\right)} \quad \text { if } m=n
$$

and

$$
\mathbf{E}\left[I_{n}(f) I_{m}(g)\right]=0 \quad \text { if } m \neq n .
$$

It also holds that

$$
I_{n}(f)=I_{n}(\tilde{f})
$$

where $\tilde{f}$ denotes the symmetrization of $f$ defined by $\tilde{f}\left(x_{1}, \ldots, x_{n}\right)=$ $(1 / n !) \sum_{\sigma \in S_{n}} f\left(x_{\sigma(1)}, \ldots, x_{\sigma(n)}\right)$.

Since the set $\mathscr{S}_{n}$ is dense in $L^{2}\left([0,1]^{n}\right)$ for every $n \geqslant 1$ the mapping $I_{n}$ can be extended to an isometry from $L^{2}\left([0,1]^{n}\right)$ to $L^{2}(\Omega)$ and the above properties hold true for this extension. Note also that $I_{n}$ can be viewed as an iterated stochastic integral

$$
I_{n}(f)=n ! \int_{0}^{1} \int_{0}^{t_{n}} \cdots \int_{0}^{t_{2}} f\left(t_{1}, \ldots, t_{n}\right) d W_{t_{1}} \cdots d W_{t_{n}}
$$

here the integrals are of Itô type; this formula is easy to show for elementary $f^{\prime}$ 's, and follows for general $f \in L^{2}\left([0,1]^{n}\right)$ by a density argument.

The product for two multiple integrals can be expanded into a sum of multiple integrals (see [14]): if $f \in L^{2}\left([0,1]^{n}\right)$ and $g \in L^{2}\left([0,1]^{m}\right)$ are symmetric functions, then

$$
I_{n}(f) I_{m}(g)=\sum_{l=0}^{m \wedge n} l ! C_{m}^{l} C_{n}^{l} I_{m+n-2 l}\left(f \otimes_{l} g\right)
$$

where the contraction $f \otimes_{l} g$ belongs to $L^{2}\left([0,1]^{m+n-2 l}\right)$ for $l=0,1, \ldots, m \wedge n$ and it is given by

$$
\begin{aligned}
&(f\left.\otimes_{l} g\right)\left(s_{1}, \ldots, s_{n-l}, t_{1}, \ldots, t_{m-l}\right) \\
& \quad=\int_{[0,1]^{l}} f\left(s_{1}, \ldots, s_{n-l}, u_{1}, \ldots, u_{l}\right) g\left(t_{1}, \ldots, t_{m-l}, u_{1}, \ldots, u_{l}\right) d u_{1} \cdots d u_{l} .
\end{aligned}
$$

When $l=0$, we use, throughout this paper, the notation $f \otimes g:=f \otimes_{0} g$. 
2.2. The Rosenblatt process. The Rosenblatt process $\left(Z^{(H)}(t)\right)_{t \in[0,1]}$ appears as a limit in the so-called noncentral limit theorem (see [7], [18], [10]). It is not a Gaussian process and can be defined through its representation as double iterated integral with respect to a standard Wiener process (see [19]). More precisely, the Rosenblatt process with self-similarity order $H \in(1 / 2,1)$ is defined by

$$
Z_{t}^{(H)}:=\int_{0}^{t} \int_{0}^{t} L_{t}\left(y_{1}, y_{2}\right) d W_{y_{1}} d W_{y_{2}},
$$

where $\left(W_{t}, t \in[0,1]\right)$ is a Brownian motion,

$$
\begin{aligned}
L_{t}^{(H)}\left(y_{1}, y_{2}\right) & :=L_{t}\left(y_{1}, y_{2}\right) \\
& =d(H) 1_{[0, t]}\left(y_{1}\right) 1_{[0, t]}\left(y_{2}\right) \int_{y_{1} \vee y_{2}}^{t} \frac{\partial K^{H^{\prime}}}{\partial u}\left(u, y_{1}\right) \frac{\partial K^{H^{\prime}}}{\partial u}\left(u, y_{2}\right) d u,(6)
\end{aligned}
$$

with

$$
H^{\prime}:=\frac{H+1}{2} \quad \text { and } \quad d(H):=\frac{1}{H+1}\left(\frac{H}{2(2 H-1)}\right)^{-1 / 2}
$$

and with $K^{H}$ the standard kernel appearing in the Wiener integral representation of the fractional Brownian motion (for $t>s$ and $H>1 / 2$ ):

$$
K^{H}(t, s):=c_{H} s^{1 / 2-H} \int_{s}^{t}(u-s)^{H-3 / 2} u^{H-1 / 2} d u,
$$

where $c_{H}=(H(2 H-1) / \beta(2-2 H, H-1 / 2))^{1 / 2}$ and $\beta(\cdot, \cdot)$ is the beta function. The derivative of $K^{H}$ is

$$
\frac{\partial K^{H}}{\partial t}(t, s)=: \partial_{1} K^{H}(t, s)=c_{H}\left(\frac{s}{t}\right)^{1 / 2-H}(t-s)^{H-3 / 2} .
$$

The two-parameter function $L_{t}$ given by (6) will be called the kernel of the Rosenblatt process. The following key relation is crucial in our calculation and will be used repeatedly in the paper:

$$
\int_{0}^{u \wedge v} \partial_{1} K^{H^{\prime}}(u, y) \partial_{1} K^{H^{\prime}}(v, y) d y=a(H)|u-v|^{2 H^{\prime}-2}
$$

with $a(H)=H^{\prime}\left(2 H^{\prime}-1\right)$. Recall several main properties of the Rosenblatt process:

(a) it is $H$-self-similar in the sense that for any $c>0$, we have $\left(Z_{c t}^{(H)}\right)={ }^{(d)}\left(c^{H} Z_{t}^{(H)}\right)$, where $=^{(d)}$ means equivalence of all finite dimensional distributions;

(b) it has stationary increments, that is, the joint distribution of $\left(Z_{t+h}^{(H)}-\right.$ $\left.Z_{h}^{(H)}, t \in[0,1]\right)$ is independent of $h>0$;

(c) $\mathbf{E}\left|Z_{t}^{(H)}\right|^{p}<\infty$ for any $p>0$, and $Z^{(H)}$ has the same variance and covariance that a standard fractional Brownian motion with parameter $H$;

(d) the Rosenblatt process is Hölder continuous, of order $\delta<H$; this can easily be obtained by the Kolmogorov continuity criterion. 
3. Renormalization of the cubic variation.

3.1. Estimation of the mean square. We will study in this paper the cubic variation of the Rosenblatt process obtained by putting $p=3$ in (1):

$$
V^{3, N}=\frac{1}{N} \sum_{i=0}^{N-1}\left(\frac{\left(Z_{(i+1) / N}^{(H)}-Z_{i / N}^{(H)}\right)^{3}}{\mathbf{E}\left(Z_{(i+1) / N}^{(H)}-Z_{i / N}^{(H)}\right)^{3}}-1\right) .
$$

Note that this expression is immaterial in the case of the fractional Brownian motion because the third moment of a centered Gaussian random variable is zero. By denoting for $=1, \ldots, N$

$$
f_{i, N}=L_{(i+1) / N}^{(H)}-L_{i / N}^{(H)}
$$

we obtain $Z_{(i+1) / N}^{(H)}-Z_{i / N}^{(H)}=I_{2}\left(f_{i, N}\right)$, where $I_{2}$ is a multiple integral of order 2 as defined in Subsection 2.1, and then

$$
V^{3, N}=\frac{1}{N} \sum_{i=0}^{N-1}\left(\frac{\left(I_{2}\left(f_{i, N}\right)\right)^{3}}{\mathbf{E}\left(I_{2}\left(f_{i, N}\right)\right)^{3}}-1\right) .
$$

By using the product formula for multiple Wiener-Itô integrals (3), for any symmetric function $f \in L^{2}\left([0,1]^{2}\right)$, we obtain

$$
\begin{aligned}
\left(I_{2}(f)\right)^{3}= & I_{6}((f \widetilde{\otimes} f) \otimes f)+8 I_{4}\left((f \widetilde{\otimes} f) \otimes_{1} f\right)+4 I_{4}\left(\left(f \otimes_{1} f\right) \otimes f\right) \\
& +12 I_{2}\left((f \widetilde{\otimes} f) \otimes_{2} f\right)+16 I_{2}\left(\left(f \otimes_{1} f\right) \otimes_{1} f\right)+2\langle f, f\rangle_{L^{2}\left([0,1]^{2}\right)} I_{2}(f) \\
& +8\left\langle\left(f \otimes_{1} f\right), f\right\rangle_{L^{2}\left([0,1]^{2}\right)} .
\end{aligned}
$$

Here and in the sequel $f \widetilde{\otimes} f$ denotes the symmetrization of the function $f \otimes f$ which is not necessarily symmetric even if $f$ is symmetric. Applying this to $f=f_{i, N}$ we obtain

$$
\left(I_{2}\left(f_{i, N}\right)\right)^{3}=8\left(f_{i, N} \otimes_{1} f_{i, N}\right) \otimes_{2} f_{i, N}+I_{2}\left(g_{i, N}\right)+4 I_{4}\left(h_{i, N}\right)+I_{6}\left(\left(f_{i, N} \widetilde{\otimes} f_{i, N}\right) \otimes f_{i, N}\right),
$$

where

$$
\begin{aligned}
g_{i, N} & =2\left\|f_{i, N}\right\|_{L^{2}}^{2} f_{i, N}+12\left(f_{i, N} \widetilde{\otimes} f_{i, N}\right) \otimes_{2} f_{i, N}+16\left(f_{i, N} \otimes_{1} f_{i, N}\right) \otimes_{1} f_{i, N}, \\
h_{i, N} & =2\left(f_{i, N} \widetilde{\otimes} f_{i, N}\right) \otimes_{1} f_{i, N}+f_{i, N} \otimes\left(f_{i, N} \otimes_{1} f_{i, N}\right) .
\end{aligned}
$$

Note that $g_{i, N} \in L^{2}\left([0,1]^{2}\right)$ and $h_{i, N} \in L^{2}\left([0,1]^{4}\right)$. On the other hand, we can simplify a little bit the above expressions, since

$$
\left(f_{i, N} \widetilde{\otimes} f_{i, N}\right) \otimes_{2} f_{i, N}=\frac{1}{3}\left\|f_{i, N}\right\|_{L^{2}}^{2} f_{i, N}+\frac{2}{3}\left(f_{i, N} \otimes_{1} f_{i, N}\right) \otimes_{1} f_{i, N} .
$$

Hence the kernel of the second chaos term can be written as

$$
g_{i, N}=6\left\|f_{i, N}\right\|_{L^{2}}^{2} f_{i, N}+24\left(f_{i, N} \otimes_{1} f_{i, N}\right) \otimes_{1} f_{i, N} .
$$


We start with the following lemma, where we compute the cubic mean of the increment of the Rosenblatt process. We already observed a significant difference from the Gaussian case: this cubic mean is not zero.

Lemma 1. Let $\left(Z_{t}^{(H)}\right)_{t \in[0,1]}$ be a Rosenblatt process with self-similarity index $H \in(1 / 2,1)$. Then, for every $s, t \in[0,1]$

$$
\mathbf{E}\left(Z_{t}^{(H)}-Z_{s}^{(H)}\right)^{3}=C(H)|t-s|^{3 H},
$$

where

$$
C(H)=8 a(H)^{3} d(H)^{3} \int_{[0,1]^{3}}\left(\left|u-v \| u-u^{\prime}\right|\left|v-u^{\prime}\right|\right)^{2 H^{\prime}-2} d u d u^{\prime} d v .
$$

P r o o f. Let us denote

$$
f_{s, t}(x, y)=L_{t}(x, y)-L_{s}(x, y)
$$

where $L$ is the kernel of the Rosenblatt process given by (6) and $x, y \in[0,1]$. We have, by using relation (9),

$$
\begin{aligned}
\left(f_{s, t}\right. & \left.\otimes_{1} f_{s, t}\right)(x, y)=\int_{0}^{1} f_{s, t}(x, z) f_{s, t}(y, z) d z \\
= & d(H)^{2} a(H)\left(1_{[0, t]}(x, y) \int_{x}^{t} \int_{y}^{t} \partial_{1} K^{H^{\prime}}(u, x) \partial_{1} K^{H^{\prime}}(v, y)|u-v|^{2 H^{\prime}-2} d v d u\right. \\
& -1_{[0, t]}(x) 1_{[0, s]}(y) \int_{x}^{t} \int_{y}^{s} \partial_{1} K^{H^{\prime}}(u, x) \partial_{1} K^{H^{\prime}}(v, y)|u-v|^{2 H^{\prime}-2} d v d u \\
& -1_{[0, s]}(x) 1_{[0, t]}(y) \int_{x}^{s} \int_{y}^{t} \partial_{1} K^{H^{\prime}}(u, x) \partial_{1} K^{H^{\prime}}(v, y)|u-v|^{2 H^{\prime}-2} d v d u \\
& \left.+1_{[0, s]}(x, y) \int_{x}^{s} \int_{y}^{s} \partial_{1} K^{H^{\prime}}(u, x) \partial_{1} K^{H^{\prime}}(v, y)|u-v|^{2 H^{\prime}-2} d v d u\right) .
\end{aligned}
$$

The computation of the cubic mean of a multiple integral in the second chaos (11) implies

$$
\mathbf{E}\left(Z_{t}^{(H)}-Z_{s}^{(H)}\right)^{3}=8\left\langle f_{s, t} \otimes_{1} f_{s, t}, f_{s, t}\right\rangle_{L^{2}\left([0,1]^{2}\right)} .
$$

We compute, by (9):

$$
\begin{aligned}
\left\langle f_{s, t}\right. & \left.\otimes_{1} f_{s, t}, f_{s, t}\right\rangle_{L^{2}\left([0,1]^{2}\right)}=\int_{[0,1]^{2}}\left(f_{s, t} \otimes_{1} f_{s, t}\right)(x, y) f_{s, t}(x, y) d x d y \\
& =d(H)^{3} a(H)^{3} \int_{s}^{t} \int_{s}^{t} \int_{s}^{t}\left(\left|u-v \| u-u^{\prime}\right|\left|v-u^{\prime}\right|\right)^{2 H^{\prime}-2} d u d u^{\prime} d v .
\end{aligned}
$$

By the change of variables $\bar{u}=(u-s) /(t-s)$ we will transform the integrals on $[s, t]$ into integrals from 0 to 1 . We immediately obtain the relation (14). Lemma 1 is proved. 
To calculate $\mathbf{E}\left(V^{3, N}\right)^{2}$ we apply the above result and we obtain

$$
\begin{aligned}
& \mathbf{E}\left(I_{2}\left(f_{i, N}\right)\right)^{3}=8\left(f_{i, N} \otimes_{1} f_{i, N}\right) \otimes_{2} f_{i, N} \\
& \quad=8 d(H)^{3} a(H)^{3} \int_{I_{i}} \int_{I_{i}} \int_{I_{i}} d y_{1} d y_{2} d y_{3}\left(\left|y_{1}-y_{2}\right|\left|y_{2}-y_{3}\right|\left|y_{3}-y_{1}\right|\right)^{2 H^{\prime}-2} \\
& \quad=8 \frac{d(H)^{3} a(H)^{3}}{N^{6 H^{\prime}-3}} \int_{[0,1]^{3}} d y_{1} d y_{2} d y_{3}\left(\left|y_{1}-y_{2}\right|\left|y_{2}-y_{3}\right|\left|y_{3}-y_{1}\right|\right)^{2 H^{\prime}-2} \\
& \quad=C(H) N^{-\left(6 H^{\prime}-3\right)}=C(H) N^{-3 H},
\end{aligned}
$$

where $I_{i}=[i / N,(i+1) / N], a(H)=H(H+1) / 2$, and $C(H)$ is defined in (15).

We can write the expression of the statistics $V^{3, N}$ as follows:

$$
\begin{aligned}
V^{3, N}= & \frac{1}{C(H) N^{1-3 H}} \sum_{i=0}^{N-1}\left(\left(I_{2}\left(f_{i, N}\right)\right)^{3}-\mathbf{E}\left(I_{2}\left(f_{i, N}\right)\right)^{3}\right)=\frac{1}{C(H) N^{1-3 H}} \\
& \times \sum_{i=0}^{N-1}\left(I_{2}\left(g_{i, N}\right)+4 I_{4}\left(h_{i, N}\right)+I_{6}\left(\left(f_{i, N} \widetilde{\otimes} f_{i, N}\right) \otimes f_{i, N}\right)\right) .
\end{aligned}
$$

We prove next the following renormalization result.

Proposition 1. Let $V^{3, N}$ be the cubic variation statistics of the Rosenblatt process. Then

$$
\mathbf{E}\left(N^{1-H} V^{3, N}\right)^{2} \longrightarrow \bar{C}(H), \quad N \rightarrow \infty,
$$

where $\widetilde{C}(H):=C(H)^{2} C_{0}(H)$ with

$$
C_{0}(H)=9+36 C^{\prime}(H) H(2 H-1)+144\left[C^{\prime}(H) H(2 H-1)\right]^{2} .
$$

P r o o f. The isometry property of multiple Wiener-Itô integrals and relation (16) imply

$$
\begin{aligned}
& \mathbf{E}\left(V^{3, N}\right)^{2}= \frac{1}{N^{2}\left(\mathbf{E}\left(I_{2}\left(f_{i, N}\right)\right)^{3}\right)^{2}} \\
& \times \sum_{i, j=0}^{N-1}\left[\mathbf{E}\left(I_{2}\left(g_{i, N}\right) I_{2}\left(g_{j, N}\right)\right)+16 \mathbf{E}\left(I_{4}\left(h_{i, N}\right) I_{4}\left(h_{j, N}\right)\right)\right. \\
&\left.\quad+\mathbf{E}\left(I_{6}\left(\left(f_{i, N} \widetilde{\otimes} f_{i, N}\right) \otimes f_{i, N}\right) I_{6}\left(\left(f_{j, N} \widetilde{\otimes} f_{j, N}\right) \otimes f_{j, N}\right)\right)\right] \\
&=\frac{1}{C(H)^{2} N^{2-6 H}}\left[\sum_{i, j=0}^{N-1} 2 !\left\langle g_{i, N}, g_{j, N}\right\rangle_{L^{2}\left([0,1]^{2}\right)}+\sum_{i, j=0}^{N-1} 4 ! \cdot 16\left\langle\widetilde{h}_{i, N}, \widetilde{h}_{j, N}\right\rangle_{L^{2}\left([0,1]^{4}\right)}\right. \\
&\left.\quad+\sum_{i, j=0}^{N-1} 6 !\left\langle\left(f_{i, N} \widetilde{\otimes} f_{i, N}\right) \otimes f_{i, N},\left(f_{j, N} \widetilde{\otimes} f_{j, N}\right) \otimes f_{j, N}\right\rangle_{L^{2}\left([0,1]^{6}\right)}\right] \\
&=: \frac{1}{C(H)^{2} N^{2-6 H}}\left[A_{N}^{(2)}+B_{N}^{(4)}+D_{N}^{(6)}\right] .
\end{aligned}
$$


We use the notation $A_{N}^{(2)}$ to indicate that this term comes from the estimation of the second chaos summand of $V^{3, N}$, and similarly for the terms $B_{N}^{(4)}$ and $D_{N}^{(6)}$. We will try to estimate all the three terms above to see which is the dominant term.

Estimation of the term $A_{N}^{(2)}$. Taking into account the expression of the second chaos kernel $g_{i, N}(12)$, we get

$$
\begin{aligned}
& A_{N}^{(2)}=2 \sum_{i, j=0}^{N-1}[ 36\left\|f_{i, N}\right\|_{L^{2}\left([0,]^{2}\right)}^{2}\left\|f_{j, N}\right\|_{L^{2}\left([0,1]^{2}\right)}^{2}\left\langle f_{i, N}, f_{j, N}\right\rangle_{L^{2}\left([0,1]^{2}\right)} \\
&+144\left\|f_{i, N}\right\|_{L^{2}\left([0,1]^{2}\right)}^{2}\left\langle f_{i, N},\left(f_{j, N} \otimes_{1} f_{j, N}\right) \otimes_{1} f_{j, N}\right\rangle_{L^{2}\left([0,1]^{2}\right)} \\
&\left.+(24)^{2}\left\langle\left(f_{i, N} \otimes_{1} f_{i, N}\right) \otimes_{1} f_{i, N},\left(f_{j, N} \otimes_{1} f_{j, N}\right) \otimes_{1} f_{j, N}\right\rangle_{L^{2}\left([0,1]^{2}\right)}\right] \\
&=: 2\left(36 A_{1, N}^{(2)}+144 A_{2, N}^{(2)}+(24)^{2} A_{3, N}^{(2)}\right) .
\end{aligned}
$$

Let us evaluate the term $A_{1, N}^{(2)}$. We have

$$
\begin{aligned}
2 !\left\|f_{i, N}\right\|_{L^{2}\left([0,1]^{2}\right)}^{2} & =\mathbf{E}\left|Z_{(i+1) / N}^{(H)}-Z_{i / N}^{(H)}\right|^{2}=N^{-2 H}, \\
2\left\langle f_{i, N}, f_{j, N}\right\rangle_{L^{2}\left([0,1]^{2}\right)} & =\mathbf{E}\left(Z_{(i+1) / N}^{(H)}-Z_{i / N}^{(H)}\right)\left(Z_{(j+1) / N}^{(H)}-Z_{j / N}^{(H)}\right) .
\end{aligned}
$$

Hence

$$
\begin{aligned}
A_{1, N}^{(2)} & =\sum_{i, j=0}^{N-1}\left[\left\|f_{i, N}\right\|_{L^{2}\left([0,1]^{2}\right)}^{2}\left\|f_{j, N}\right\|_{L^{2}\left([0,1]^{2}\right)}^{2}\left\langle f_{i, N}, f_{j, N}\right\rangle_{L^{2}\left([0,1]^{2}\right)}\right] \\
& =\frac{1}{8} N^{-4 H} \sum_{i, j=0}^{N-1} \mathbf{E}\left(Z_{(i+1) / N}^{(H)}-Z_{i / N}^{(H)}\right)\left(Z_{(j+1) / N}^{(H)}-Z_{j / N}^{(H)}\right)=\frac{1}{8} N^{-4 H}
\end{aligned}
$$

because $\mathbf{E} \sum_{i, j=0}^{N-1}\left(Z_{(i+1) / N}^{(H)}-Z_{i / N}^{(H)}\right)\left(Z_{(j+1) / N}^{(H)}-Z_{j / N}^{(H)}\right)=\mathbf{E}\left(Z_{1}^{(H)}\right)^{2}=1$, and we have

$$
\lim _{N \rightarrow \infty} N^{4 H} A_{1, N}^{(2)}=\frac{1}{8}
$$

To evaluate $A_{3, N}^{(2)}$, note that

$$
\begin{gathered}
d(H)^{-2} a(H)^{-1}\left(f_{i, N} \otimes_{1} f_{i, N}\right)(x, y) \\
=1_{[0, i / N]}^{\otimes 2}(x, y) \int_{I_{i}} \int_{I_{i}} \partial_{1} K^{H^{\prime}}\left(u_{1}, x\right) \partial_{1} K^{H^{\prime}}\left(u_{2}, y\right)\left|u_{1}-u_{2}\right|^{2 H^{\prime}-2} d u_{2} d u_{1} \\
+1_{[0, i / N]}(x) 1_{I_{i}}(y) \int_{I_{i}} \int_{y}^{(i+1) / N} \partial_{1} K^{H^{\prime}}\left(u_{1}, x\right) \partial_{1} K^{H^{\prime}}\left(u_{2}, y\right) \\
\times\left|u_{1}-u_{2}\right|^{2 H^{\prime}-2} d u_{2} d u_{1}
\end{gathered}
$$




$$
\begin{aligned}
& +1_{[0, i / N]}(y) 1_{I_{i}}(x) \int_{x}^{(i+1) / N} \int_{I_{i}} \partial_{1} K^{H^{\prime}}\left(u_{1}, x\right) \partial_{1} K^{H^{\prime}}\left(u_{2}, y\right) \\
& \times\left|u_{1}-u_{2}\right|^{2 H^{\prime}-2} d u_{2} d u_{1} \\
& +1_{I_{i}}(x) 1_{I_{i}}(y) \int_{x}^{(i+1) / N} \int_{y}^{(i+1) / N} \partial_{1} K^{H^{\prime}}\left(u_{1}, x\right) \partial_{1} K^{H^{\prime}}\left(u_{2}, y\right) \\
& \times\left|u_{1}-u_{2}\right|^{2 H^{\prime}-2} d u_{2} d u_{1} .
\end{aligned}
$$

Sometimes the following expressions are useful:

$$
\begin{aligned}
& \left(f_{i, N} \otimes_{1} f_{i, N}\right)(x, y)=d(H)^{2} a(H) 1_{[0,(i+1) / N]}^{\otimes 2}(x, y) \\
& \quad \times \int_{I_{i}} \int_{I_{i}} \partial_{1} K^{H^{\prime}}\left(u_{1}, x\right) \partial_{1} K^{H^{\prime}}\left(u_{2}, y\right)\left|u_{1}-u_{2}\right|^{2 H^{\prime}-2} d u_{2} d u_{1}
\end{aligned}
$$

and

$$
\begin{aligned}
d(H)^{-1} f_{i, N}(x, z)= & 1_{[0, i / N]}^{\otimes 2}(x, z) \int_{I_{i}} \partial_{1} K^{H^{\prime}}\left(u_{3}, x\right) \partial_{1} K^{H^{\prime}}\left(u_{3}, z\right) d u_{3} \\
& +1_{[0, i / N]}(x) 1_{I_{i}}(z) \int_{z}^{(i+1) / N} \partial_{1} K^{H^{\prime}}\left(u_{3}, x\right) \partial_{1} K^{H^{\prime}}\left(u_{3}, z\right) d u_{3} \\
& +1_{[0, i / N]}(z) 1_{I_{i}}(x) \int_{x}^{(i+1) / N} \partial_{1} K^{H^{\prime}}\left(u_{3}, x\right) \partial_{1} K^{H^{\prime}}\left(u_{3}, z\right) d u_{3} \\
& +1_{I_{i}}(x) 1_{I_{i}}(z) \int_{I_{i}} \int_{I_{i}} \partial_{1} K^{H^{\prime}}\left(u_{3}, x\right) \partial_{1} K^{H^{\prime}}\left(u_{3}, z\right) d u_{3},
\end{aligned}
$$

or

$$
f_{i, N}(x, z)=d(H) 1_{[0,(i+1) / N]}^{\otimes 2}(x, z) \int_{I_{i}} \partial_{1} K^{H^{\prime}}\left(u_{3}, x\right) \partial_{1} K^{H^{\prime}}\left(u_{3}, z\right) d u_{3} .
$$

Therefore

$$
\begin{aligned}
& \left(\left(f_{i, N} \otimes_{1} f_{i, N}\right) \otimes_{1} f_{i, N}\right)(y, z)=d(H)^{3} a(H)^{2}\left(1_{[0,(i+1) / N]}^{\otimes 2}(y, z)\right. \\
& \left.\times \int_{I_{i}} \int_{I_{i}} \int_{I_{i}}\left(\left|u_{1}-u_{2}\right|\left|u_{1}-u_{3}\right|\right)^{2 H^{\prime}-2} \partial_{1} K^{H^{\prime}}\left(u_{2}, y\right) \partial_{1} K^{H^{\prime}}\left(u_{3}, z\right) d u_{3} d u_{2} d u_{1}\right) .
\end{aligned}
$$

The norm has a nicer expression. Using the change of variables $\bar{u}=(u-$ $i / N) N$ (which is now usual and can be used systematically) we have

$$
\begin{aligned}
\left\langle\left(f_{i, N} \otimes_{1} f_{i, N}\right) \otimes_{1}\right. & \left.f_{i, N},\left(f_{j, N} \otimes_{1} f_{j, N}\right) \otimes_{1} f_{j, N}\right\rangle_{L^{2}\left([0,1]^{2}\right)} \\
=d(H)^{6} a(H)^{6} & \int_{I_{i}^{3}} d u_{1} d u_{2} d u_{3} \int_{I_{j}^{3}} d v_{1} d v_{2} d v_{3} \\
& \times\left|u_{1}-u_{2}\right|^{2 H^{\prime}-2}\left|u_{1}-u_{3}\right|^{2 H^{\prime}-2}\left|v_{1}-v_{2}\right|^{2 H^{\prime}-2}\left|v_{1}-v_{3}\right|^{2 H^{\prime}-2} \\
& \times\left|u_{2}-v_{2}\right|^{2 H^{\prime}-2}\left|u_{3}-v_{3}\right|^{2 H^{\prime}-2} \\
=\frac{d(H)^{6} a(H)^{6}}{N^{12 H^{\prime}-6}} \int_{[0,1]^{3}} \int_{[0,1]^{3}}\left|v_{1}-v_{2}\right|^{2 H^{\prime}-2}\left|v_{2}-v_{3}\right|^{2 H^{\prime}-2} & \\
& \times\left|v_{3}-v_{4}+i-j\right|^{2 H^{\prime}-2}\left|v_{4}-v_{5}\right|^{2 H^{\prime}-2}\left|v_{5}-v_{6}\right|^{2 H^{\prime}-2} \\
& \times\left|v_{6}-v_{1}+j-i\right|^{2 H^{\prime}-2} d v_{1} \cdots d v_{6} .
\end{aligned}
$$


The rate of convergence of all terms comes actually from how many product $|u-v|^{2 H^{\prime}-2}$ with $u \in I_{i}$ and $v \in I_{j}$ we have. Hence

$$
\begin{aligned}
A_{3, N}^{(2)}=\frac{d(H)^{6} a(H)^{6}}{N^{12 H^{\prime}-6}} \sum_{i, j=1}^{N} & \int_{[0,1]^{3}} \int_{[0,1]^{3}}\left|v_{1}-v_{2}\right|^{2 H^{\prime}-2}\left|v_{2}-v_{3}\right|^{2 H^{\prime}-2} \\
& \times\left|v_{3}-v_{4}+i-j\right|^{2 H^{\prime}-2}\left|v_{4}-v_{5}\right|^{2 H^{\prime}-2}\left|v_{5}-v_{6}\right|^{2 H^{\prime}-2} \\
& \times\left|v_{6}-v_{1}+j-i\right|^{2 H^{\prime}-2} d v_{1} \cdots d v_{6} \\
=\frac{2 d(H)^{6} a(H)^{6}}{N^{12 H^{\prime}-6}} \sum_{i>}^{N} & \int_{[0,1]^{3}} \int_{[0,1]^{3}}\left|v_{1}-v_{2}\right|^{2 H^{\prime}-2}\left|v_{2}-v_{3}\right|^{2 H^{\prime}-2} \\
& \times\left|v_{3}-v_{4}+i-j\right|^{2 H^{\prime}-2}\left|v_{4}-v_{5}\right|^{2 H^{\prime}-2}\left|v_{5}-v_{6}\right|^{2 H^{\prime}-2} \\
& \times\left|v_{1}-v_{6}+i-j\right|^{2 H^{\prime}-2} d v_{1} \cdots d v_{6} \\
=\frac{2 d(H)^{6} a(H)^{6}}{N^{12 H^{\prime}-6}} \sum_{k=0}^{N-1} & (N-k) \int_{[0,1]^{3}} \int_{[0,1]^{3}}\left|v_{1}-v_{2}\right|^{2 H^{\prime}-2}\left|v_{2}-v_{3}\right|^{2 H^{\prime}-2} \\
& \times\left|v_{3}-v_{4}+k\right|^{2 H^{\prime}-2}\left|v_{4}-v_{5}\right|^{2 H^{\prime}-2}\left|v_{5}-v_{6}\right|^{2 H^{\prime}-2} \\
& \times\left|v_{1}-v_{6}+k\right|^{2 H^{\prime}-2} d v_{1} \cdots d v_{6} .
\end{aligned}
$$

We put

$$
\begin{aligned}
\bar{A}_{3, N}^{(2)} & :=\frac{1}{N^{12 H^{\prime}-6}} \sum_{k=0}^{N-1}(N-k)\left|v_{3}-v_{4}+k\right|^{2 H^{\prime}-2}\left|v_{1}-v_{6}+k\right|^{2 H^{\prime}-2} \\
& =\frac{1}{N^{8 H^{\prime}-4}} \frac{1}{N} \sum_{k=0}^{N-1}\left(1-\frac{k}{N}\right)\left|\frac{v_{3}-v_{4}}{N}+\frac{k}{N}\right|^{2 H^{\prime}-2}\left|\frac{v_{1}-v_{6}}{N}+\frac{k}{N}\right|^{2 H^{\prime}-2}
\end{aligned}
$$

and we conclude easily by a Riemann sum convergence that

$$
N^{4 H} \bar{A}_{3, N}^{(2)}=N^{8 H^{\prime}-4} \bar{A}_{3, N}^{(2)} \underset{N \rightarrow \infty}{\longrightarrow} \int_{0}^{1}(1-x) x^{4 H^{\prime}-4} d x=\frac{1}{2 H-1}-\frac{1}{2 H},
$$

because the terms $\left(v_{3}-v_{4}\right) / N$ are negligible with respect to $k / N$ for large enough $k$. This implies that

$$
N^{4 H} A_{3, N}^{(2)} \underset{N \rightarrow \infty}{\longrightarrow} \frac{d(H)^{6} a(H)^{6}}{H(2 H-1)}\left(C^{\prime}(H)\right)^{2}=\frac{H^{2}(2 H-1)^{2}}{8}\left(C^{\prime}(H)\right)^{2},
$$

where $C^{\prime}(H)=\int_{[0,1]^{3}}\left|v_{1}-v_{2}\right|^{2 H^{\prime}-2}\left|v_{2}-v_{3}\right|^{2 H^{\prime}-2} d v_{1} d v_{2} d v_{3}$. Now, we estimate the term $A_{2, N}^{(2)}$. We have

$$
\begin{aligned}
& \left\langle f_{i, N},\left(f_{j, N} \otimes_{1} f_{j, N}\right) \otimes_{1} f_{j, N}\right\rangle_{L^{2}\left([0,1]^{2}\right)} \\
& =d(H)^{4} a(H)^{4} \int_{I_{i}} \int_{I_{j}^{3}}\left|u_{1}-u_{2}\right|^{2 H^{\prime}-2}\left|u_{2}-u_{3}\right|^{2 H^{\prime}-2}\left|u_{3}-u_{4}\right|^{2 H^{\prime}-2} \\
& \quad \times\left|u_{4}-u_{1}\right|^{2 H^{\prime}-2} d u_{1} \cdots d u_{4}
\end{aligned}
$$




$$
\begin{gathered}
=\frac{d(H)^{4} a(H)^{4}}{N^{8 H^{\prime}-4}} \int_{[0,1]^{4}}\left|v_{1}-v_{2}+i-j\right|^{2 H^{\prime}-2}\left|v_{2}-v_{3}\right|^{2 H^{\prime}-2}\left|v_{3}-v_{4}\right|^{2 H^{\prime}-2} \\
\times\left|v_{4}-v_{1}+j-i\right|^{2 H^{\prime}-2} d v_{1} \cdots d v_{4} .
\end{gathered}
$$

Then

$$
\begin{aligned}
A_{2, N}^{(2)} & =\left\|f_{i, N}\right\|_{L^{2}\left([0,1]^{2}\right)}^{2} \frac{d(H)^{4} a(H)^{4}}{N^{8 H^{\prime}-4}} \int_{[0,1]^{4}}\left|v_{2}-v_{3}\right|^{2 H^{\prime}-2}\left|v_{3}-v_{4}\right|^{2 H^{\prime}-2} \\
& \times\left(\sum_{i, j=1}^{N}\left|v_{1}-v_{2}+i-j\right|^{2 H^{\prime}-2}\left|v_{4}-v_{1}+j-i\right|^{2 H^{\prime}-2}\right) d v_{1} \cdots d v_{4} \\
= & \frac{N^{-2 H}}{2} \frac{d(H)^{4} a(H)^{4}}{N^{8 H^{\prime}-4}} \int_{[0,1]^{4}}\left|v_{2}-v_{3}\right|^{2 H^{\prime}-2}\left|v_{3}-v_{4}\right|^{2 H^{\prime}-2} \\
& \times\left(2 \sum_{k=0}^{N-1}(N-k)\left|v_{1}-v_{2}+k\right|^{2 H^{\prime}-2}\left|v_{4}-v_{1}+k\right|^{2 H^{\prime}-2}\right) d v_{1} \cdots d v_{4} \\
= & N^{-2 H} \frac{d(H)^{4} a(H)^{4}}{N^{4} H^{\prime}-2} \int_{[0,1]^{4}}\left|v_{2}-v_{3}\right|^{2 H^{\prime}-2}\left|v_{3}-v_{4}\right|^{2 H^{\prime}-2} \\
& \times\left(\frac{1}{N} \sum_{k=0}^{N-1}\left(1-\frac{k}{N}\right)\left|\frac{v_{1}-v_{2}}{N}+\frac{k}{N}\right|^{2 H^{\prime}-2}\left|\frac{v_{4}-v_{1}}{N}+\frac{k}{N}\right|^{2 H^{\prime}-2}\right) d v_{1} \cdots d v_{4} \\
= & \frac{d(H)^{4} a(H)^{4}}{N^{4 H}} \int_{[0,1]^{4}}\left|v_{2}-v_{3}\right|^{2 H^{\prime}-2}\left|v_{3}-v_{4}\right|^{2 H^{\prime}-2} \\
& \times\left(\frac{1}{N} \sum_{k=0}^{N-1}\left(1-\frac{k}{N}\right)\left|\frac{v_{1}-v_{2}}{N}+\frac{k}{N}\right|^{2 H^{\prime}-2}\left|\frac{v_{4}-v_{1}}{N}+\frac{k}{N}\right|^{2 H^{\prime}-2}\right) d v_{1} \cdots d v_{4} .
\end{aligned}
$$

We obtain that $N^{4 H} A_{2, N}^{(2)}$ converges as $N \rightarrow \infty$ to

$$
\begin{aligned}
\left(\int_{0}^{1}(1-x)\right. & \left.x^{4 H^{\prime}-4} d x\right)\left(d(H)^{4} a(H)^{4}\right. \\
& \left.\times \int_{[0,1]^{3}}\left|v_{2}-v_{3}\right|^{2 H^{\prime}-2}\left|v_{3}-v_{4}\right|^{2 H^{\prime}-2} d v_{2} d v_{3} d v_{4}\right) \\
=\left(\frac{1}{2 H}-1\right. & \left.-\frac{1}{2 H}\right) d(H)^{4} a(H)^{4} \\
& \times \int_{[0,1]^{3}}\left|v_{2}-v_{3}\right|^{2 H^{\prime}-2}\left|v_{3}-v_{4}\right|^{2 H^{\prime}-2} d v_{2} d v_{3} d v_{4}
\end{aligned}
$$

Thus

$$
N^{4 H} A_{2, N}^{(2)} \underset{N \rightarrow \infty}{\longrightarrow} C^{\prime}(H) \frac{H(2 H-1)}{8} .
$$

From (18), (21), and (22), we obtain that

$$
N^{4 H} A_{N}^{(2)}=2\left(36 N^{4 H} A_{1, N}^{(2)}+144 N^{4 H} A_{2, N}^{(2)}+(24)^{2} N^{4 H} A_{3, N}^{(2)}\right)
$$


converges to $\left(9+36 C^{\prime}(H) H(2 H-1)+144\left[C^{\prime}(H) H(2 H-1)\right]^{2}\right)=: C_{0}(H)$ as $N \rightarrow \infty$. Consequently

$$
\frac{N^{4 H} A_{N}^{(2)}}{C_{0}(H)} \underset{N \rightarrow \infty}{\longrightarrow} 1
$$

Estimation of the term $D_{N}^{(6)}$. Now, we study the convergence of $D_{N}^{(6)}$. By the symmetry property of every $f_{i, N}, i=0, \ldots, N-1$, on $[0,1]^{2}$, there exist positive combinatorial constants $c_{1}, c_{2}$, and $c_{3}$ such that

$$
\begin{aligned}
& D_{N}^{(6)}=\left.\left.\sum_{i, j=0}^{N-1} 6 !\left\langle\left(f_{i, N} \widetilde{\otimes} f_{j, N}\right) \widetilde{\otimes} f_{j, N}\right),\left(f_{j, N} \widetilde{\otimes} f_{i, N}\right) \widetilde{\otimes} f_{i, N}\right)\right\rangle_{L^{2}\left([0,1]^{6}\right)} \\
&=\left.c_{1} \sum_{i, j=0}^{N-1}\left(\left\langle f_{i, N}, f_{j, N}\right\rangle_{L^{2}\left([0,1]^{2}\right)}\right)\right)^{3}+c_{2} \sum_{i, j=0}^{N-1}\left\langle f_{i, N}, f_{j, N}\right\rangle_{L^{2}\left([0,1]^{2}\right)} \\
& \times \int_{[0,1]^{4}} f_{i, N}\left(x_{1}, x_{2}\right) f_{j, N}\left(x_{2}, x_{3}\right) f_{i, N}\left(x_{3}, x_{4}\right) f_{j, N}\left(x_{4}, x_{1}\right) d x_{1} \cdots d x_{4} \\
&+c_{3} \sum_{i, j=0}^{N-1} \int_{[0,1]^{6}} f_{i, N}\left(x_{1}, x_{2}\right) f_{j, N}\left(x_{2}, x_{3}\right) f_{i, N}\left(x_{3}, x_{4}\right) f_{j, N}\left(x_{4}, x_{5}\right) \\
& \quad \times f_{i, N}\left(x_{5}, x_{6}\right) f_{j, N}\left(x_{6}, x_{1}\right) d x_{1} \cdots d x_{6} \\
&=: c_{1} D_{1, N}^{(6)}+c_{2} D_{2, N}^{(6)}+c_{3} D_{3, N}^{(6)} .
\end{aligned}
$$

By using the same argument as above, we obtain

$$
\begin{aligned}
D_{1, N}^{(6)} & =\sum_{i, j=0}^{N-1}\left(\left\langle f_{i, N}, f_{j, N}\right\rangle_{L^{2}\left([0,1]^{2}\right)}\right)^{3} \\
& =\frac{d(H)^{6} a(H)^{6}}{N^{6 H}} \sum_{i, j=0}^{N-1}\left(\int_{[0,1]^{2}}\left|x_{1}-x_{2}+i-j\right|^{2 H-2} d x_{1} d x_{2}\right)^{3} \\
& =\frac{d(H)^{6} a(H)^{6}}{N^{6 H}} 2 \sum_{k=0}^{N-1}(N-k)\left(\int_{[0,1]^{2}}\left|x_{1}-x_{2}+k\right|^{2 H-2} d x_{1} d x_{2}\right)^{3} \\
& =\frac{2 d(H)^{6} a(H)^{6}}{N^{4}} \sum_{k=0}^{N-1} \frac{1}{N}\left(1-\frac{k}{N}\right)\left(\int_{[0,1]^{2}}\left|\frac{x_{1}-x_{2}}{N}+\frac{k}{N}\right|^{2 H-2} d x_{1} d x_{2}\right)^{3},
\end{aligned}
$$

and clearly, since $H<1$, we have

$$
\lim _{N \rightarrow \infty} N^{4 H} D_{1, N}^{(6)}=0
$$

In the same way, we obtain

$$
\begin{aligned}
D_{2, N}^{(6)}= & \sum_{i, j=0}^{N-1}\left\langle f_{i, N}, f_{j, N}\right\rangle_{L^{2}\left([0,1]^{2}\right)} \\
& \times \int_{[0,1]^{4}} f_{i, N}\left(x_{1}, x_{2}\right) f_{j, N}\left(x_{2}, x_{3}\right) f_{i, N}\left(x_{3}, x_{4}\right) f_{j, N}\left(x_{4}, x_{1}\right) d x_{1} \cdots d x_{4}
\end{aligned}
$$




$$
\begin{aligned}
& =\sum_{i, j=0}^{N-1} N^{-4} d(H)^{4} a(H)^{4}\left\langle f_{i, N}, f_{j, N}\right\rangle_{L^{2}\left([0,1]^{2}\right)} \int_{[0,1]^{4}}\left|\frac{x_{1}-x_{2}}{N}+\frac{i-j}{N}\right|^{2 H^{\prime}-2} \\
& \times\left|\frac{x_{3}-x_{2}}{N}+\frac{i-j}{N}\right|^{2 H^{\prime}-2}\left|\frac{x_{3}-x_{4}}{N}+\frac{i-j}{N}\right|^{2 H^{\prime}-2} \\
& \times\left|\frac{x_{1}-x_{4}}{N}+\frac{i-j}{N}\right|^{2 H^{\prime}-2} d x_{1} \cdots d x_{4} \\
& =\frac{2 d(H)^{6} a(H)^{6}}{N^{6}} \sum_{k=0}^{N-1}(N-k) \int_{[0,1]^{2}}\left|\frac{x_{5}-x_{6}}{N}+\frac{k}{N}\right|^{2 H-2} d x_{5} d x_{6} \\
& \times \int_{[0,1]^{4}}\left|\frac{x_{1}-x_{2}}{N}+\frac{k}{N}\right|^{2 H^{\prime}-2}\left|\frac{x_{3}-x_{2}}{N}+\frac{k}{N}\right|^{2 H^{\prime}-2}\left|\frac{x_{3}-x_{4}}{N}+\frac{k}{N}\right|^{2 H^{\prime}-2} \\
& \times\left|\frac{x_{1}-x_{4}}{N}+\frac{k}{N}\right|^{2 H^{\prime}-2} d x_{1} \cdots d x_{4} \\
& =\frac{2 d(H)^{6} a(H)^{6}}{N^{4}} \sum_{k=0}^{N-1} \frac{1}{N}\left(1-\frac{k}{N}\right) \int_{[0,1]^{2}}\left|\frac{x_{5}-x_{6}}{N}+\frac{k}{N}\right|^{2 H-2} d x_{5} d x_{6} \\
& \times \int_{[0,1]^{4}}\left|\frac{x_{1}-x_{2}}{N}+\frac{k}{N}\right|^{2 H^{\prime}-2}\left|\frac{x_{3}-x_{2}}{N}+\frac{k}{N}\right|^{2 H^{\prime}-2}\left|\frac{x_{3}-x_{4}}{N}+\frac{k}{N}\right|^{2 H^{\prime}-2} \\
& \times\left|\frac{x_{1}-x_{4}}{N}+\frac{k}{N}\right|^{2 H^{\prime}-2} d x_{1} \cdots d x_{4} .
\end{aligned}
$$

This implies that

$$
\lim _{N \rightarrow \infty} N^{4 H} D_{2, N}^{(6)}=0
$$

In the same manner as in previous results, we have

$$
\begin{aligned}
D_{3, N}^{(6)}= & \sum_{i, j=0}^{N-1} \int_{[0,1]^{6}} f_{i, N}\left(x_{1}, x_{2}\right) f_{j, N}\left(x_{2}, x_{3}\right) f_{i, N}\left(x_{3}, x_{4}\right) f_{j, N}\left(x_{4}, x_{5}\right) \\
& \times f_{i, N}\left(x_{5}, x_{6}\right) f_{j, N}\left(x_{6}, x_{1}\right) d x_{1} \ldots d x_{6} \\
= & \frac{d(H)^{6} a(H)^{6}}{N^{6}} \sum_{i, j=0}^{N-1} \int_{[0,1]^{6}}\left|\frac{x_{1}-x_{2}}{N}+\frac{i-j}{N}\right|^{2 H^{\prime}-2}\left|\frac{x_{3}-x_{2}}{N}+\frac{i-j}{N}\right|^{2 H^{\prime}-2} \\
\times & \left|\frac{x_{3}-x_{4}}{N}+\frac{i-j}{N}\right|^{2 H^{\prime}-2}\left|\frac{x_{5}-x_{4}}{N}+\frac{i-j}{N}\right|^{2 H^{\prime}-2} \\
\times & \left|\frac{x_{5}-x_{6}}{N}+\frac{i-j}{N}\right|^{2 H^{\prime}-2}\left|\frac{x_{1}-x_{6}}{N}+\frac{i-j}{N}\right|^{2 H^{\prime}-2} d x_{1} \cdots d x_{6} \\
= & \frac{2 d(H)^{6} a(H)^{6}}{N^{4}} \frac{1}{N-1}\left(1-\frac{k}{N}\right) \int_{[0,1]^{4}} \frac{x_{1}-x_{2}}{N}+\left.\frac{k}{N}\right|^{2 H^{\prime}-2} \\
\times & \left|\frac{x_{3}-x_{2}}{N}+\frac{k}{N}\right|^{2 H^{\prime}-2}\left|\frac{x_{3}-x_{4}}{N}+\frac{k}{N}\right|^{2 H^{\prime}-2}
\end{aligned}
$$




$$
\begin{aligned}
& \times\left|\frac{x_{5}-x_{4}}{N}+\frac{k}{N}\right|^{2 H^{\prime}-2}\left|\frac{x_{5}-x_{6}}{N}+\frac{k}{N}\right|^{2 H^{\prime}-2} \\
& \times\left|\frac{x_{1}-x_{6}}{N}+\frac{k}{N}\right|^{2 H^{\prime}-2} d x_{1} \cdots d x_{6} .
\end{aligned}
$$

Hence

$$
\lim _{N \rightarrow \infty} N^{4 H} D_{3, N}^{(6)}=0
$$

Thus, from (25)-(27), it follows that

$$
\lim _{N \rightarrow \infty} N^{4 H} D_{N}^{(6)}=0
$$

Estimation of the term $B_{N}^{(4)}$. The same argument as above yields that there exist constants $c_{1}^{\prime}$ and $c_{2}^{\prime}$ such that

$$
\begin{aligned}
& \left\langle\widetilde{h}_{i, N}, \widetilde{h}_{j, N}\right\rangle_{L^{2}\left([0,1]^{4}\right)} \\
& =c_{1}^{\prime} \sum_{i, j=0}^{N-1}\left\langle f_{i, N}, f_{j, N}\right\rangle_{L^{2}\left([0,]^{2}\right)} \\
& \quad \times \int_{[0,1]^{4}} f_{i, N}\left(x_{1}, x_{2}\right) f_{j, N}\left(x_{2}, x_{3}\right) f_{i, N}\left(x_{3}, x_{4}\right) f_{j, N}\left(x_{4}, x_{1}\right) d x_{1} \ldots d x_{4} \\
& \quad+c_{2}^{\prime} \sum_{i, j=0}^{N-1} \int_{[0,1]^{6}} f_{i, N}\left(x_{1}, x_{2}\right) f_{j, N}\left(x_{2}, x_{3}\right) f_{i, N}\left(x_{3}, x_{4}\right) f_{j, N}\left(x_{4}, x_{5}\right) \\
& \quad \times f_{i, N}\left(x_{5}, x_{6}\right) f_{j, N}\left(x_{6}, x_{1}\right) d x_{1} \cdots d x_{6} \\
& =c_{1}^{\prime} D_{2, N}^{(6)}+c_{2}^{\prime} D_{3, N}^{(6)} .
\end{aligned}
$$

The same terms as in the estimation of the sixth chaos kernel appear. Thus, from the convergences (26) and (27),

$$
\begin{aligned}
\lim _{N \rightarrow \infty} N^{4 H} B_{N}^{(4)} & =\lim _{N \rightarrow \infty} N^{4 H} \sum_{i, j=0}^{N-1}\left\langle\widetilde{h}_{i, N}, \widetilde{h}_{j, N}\right\rangle_{L^{2}\left([0,1]^{4}\right)} \\
& =\lim _{N \rightarrow \infty}\left(c_{1}^{\prime} N^{4 H} D_{2, N}^{(6)}+c_{2}^{\prime} N^{4 H} D_{3, N}^{(6)}\right)=0 .
\end{aligned}
$$

As a consequence of the convergences (24), (28), and (29), we have proved that for every $H>1 / 2$ and with the notation $\bar{C}(H)=C(H)^{2} / C_{0}(H)$,

$$
\frac{C(H)^{2}}{C_{0}(H)} N^{2-2 H} \mathbf{E}\left(V^{3, N}\right)^{2}=\mathbf{E}\left(\sqrt{\bar{C}(H)} N^{1-H} V^{3, N}\right)^{2} \underset{N \rightarrow \infty}{\longrightarrow} 1 .
$$

Proposition 1 is proved. 
3.2. Nonconvergence to a Gaussian limit. We prove that the normalized variation does not converge in distribution to the normal law. Of course, this is somehow superfluous taking into account that in the next section we show a noncentral limit theorem for this statistics, but we found the calculations instructive to see why it does not converges to a Gaussian limit. Recall that by $\left[15\right.$, Theorem ] a sequence $F_{N}=I_{q}\left(f_{N}\right)$ in the $q$ Wiener chaos with $\mathbf{E} F_{N}^{2} \rightarrow 1, N \rightarrow \infty$, converges to the normal law $N(0,1)$ if and only if $\left\|D F_{N}\right\|_{L^{2}[0,1]}^{2}$ converges to $q$ in $L^{2}(\Omega)$ as $N \rightarrow \infty$. Here $D$ denotes the Malliavin derivative; if $f \in L^{2}\left([0, T]^{n}\right)$ is a symmetric function, we will use the following rule to differentiate in the Malliavin sense:

$$
D_{t} I_{n}(f)=n I_{n-1}(f(\cdot, t)), \quad t \in[0,1] .
$$

We put

$$
T_{N}=\frac{\sqrt{\bar{C}(H)} N^{1-H}}{C(H) N^{1-3 H}} \sum_{i=0}^{N} I_{2}\left(g_{i, N}\right)=\frac{N^{2 H}}{\sqrt{C_{0}(H)}} \sum_{i=0}^{N} I_{2}\left(g_{i, N}\right) .
$$

We derive $T_{N}$ in the Malliavin sense and we obtain $D_{t} T_{N}=$ $\left(2 N^{2 H} / \sqrt{\left.C_{0}(H)\right)} \sum_{i=0}^{N} I_{1}\left(g_{i, N}(\cdot, t)\right)\right.$ and thus

$$
\begin{aligned}
& \left\|D T_{N}\right\|_{L^{2}\left([0,1]^{2}\right)}^{2}=\frac{4 N^{4 H}}{C_{0}(H)} \int_{0}^{1}\left(\sum_{i=0}^{N} I_{1}\left(g_{i, N}(\cdot, t)\right)\right)^{2} d t \\
& =\frac{4 N^{4 H}}{C_{0}(H)} \int_{0}^{1}\left(\sum_{i, j=0}^{N} I_{1}\left(g_{i, N}(\cdot, t)\right) I_{1}\left(g_{j, N}(\cdot, t)\right)\right) d t \\
& \quad=\frac{4 N^{4 H}}{C_{0}(H)}\left(\sum_{i, j=0}^{N} \int_{0}^{1} I_{0}\left(g_{i, N} \otimes_{1} g_{j, N}\right) d t+\sum_{i, j=0}^{N} \int_{0}^{1} I_{2}\left(g_{i, N} \otimes_{0} g_{j, N}\right) d t\right) \\
& =: \frac{4 N^{4 H}}{C_{0}(H)}\left(J_{1, N}+J_{2, N}\right),
\end{aligned}
$$

where

$$
J_{1, N}:=\sum_{i, j=0}^{N} \int_{0}^{1} I_{0}\left(g_{i, N} \otimes_{1} g_{j, N}\right) d t=\sum_{i, j=0}^{N}\left\langle g_{i, N}, g_{j, N}\right\rangle_{L^{2}\left([0,1]^{2}\right)}=\frac{1}{2} A_{N}^{(2)} .
$$

From (24) we obtain

$$
\frac{4 N^{4 H}}{C_{0}(H)} J_{1, N} \underset{N \rightarrow \infty}{\longrightarrow} 2
$$

in $L^{2}(\Omega)$ because the term $A_{N}^{(2)}$ is deterministic. To prove that $\left\|D T_{N}\right\|_{L^{2}\left([0,1]^{2}\right)}^{2}$ does not converge in $L^{2}(\Omega)$ to 2 , it is sufficient to show that

$$
\lim _{N \rightarrow \infty} \mathbf{E}\left(\frac{4 N^{4 H}}{C_{0}(H)} J_{2, N}\right)^{2}>0 .
$$


where $J_{2, N}=\sum_{i, j=0}^{N} \int_{0}^{1} I_{2}\left(g_{i, N}(\cdot, t) g_{j, N}(\cdot, t)\right) d t$.

We calculate the mean square of this term:

$$
\begin{aligned}
\mathbf{E} & \left(J_{2, N}\right)^{2}=2 \int_{[0,1]^{2}}\left(\sum_{i, j=0}^{N} \int_{0}^{1} I_{2}\left(g_{i, N}(r, t) g_{j, N}(s, t)\right) d t\right)^{2} d r d s \\
= & 2 \sum_{i, j, k, l=0}^{N} \int_{[0,1]^{4}} g_{i, N}(r, t) g_{j, N}(s, t) g_{k, N}(r, u) g_{l, N}(s, u) d r d s d t d u \\
\geqslant & \sum_{i, j, k, l=0}^{N} \int_{[0,1]^{4}} f_{i, N}(r, t) f_{j, N}(s, t) f_{k, N}(r, u) f_{l, N}(s, u) d r d s d t d u \\
= & 2 d(H)^{4} a(H)^{4} N^{-8 H} \\
& \times \sum_{i, j, k, l=0}^{N} \int_{I_{i}} \int_{I_{j}} \int_{I_{k}} \int_{I_{l}}(|r-t||s-t||r-u \| s-u|)^{2 H^{\prime}-2} d r d s d t d u \\
= & 2 d(H)^{4} a(H)^{4} N^{-8 H} \sum_{i, j, k, l=0}^{N} \int_{[0,1]^{4}} d r d s d t d u \\
& \times \frac{1}{N^{4}}\left(\left|\frac{r-t+i-j}{N}\right|\left|\frac{t-s+j-k}{N} \| \frac{s-u+k-l}{N}\right|\right. \\
& \left.\times\left|\frac{u-r+l-i}{N}\right|\right)^{2 H^{\prime}-2} .
\end{aligned}
$$

By using Riemann sums approximations, we obtain

$$
\begin{aligned}
\lim _{N \rightarrow \infty} \mathbf{E}\left(N^{4 H} J_{2, N}\right)^{2} \geqslant & 2 d(H)^{4} a(H)^{4} \int_{[0,1]^{4}} d x_{1} d x_{2} d x_{3} d x_{4} \\
& \times\left(\left|x_{1}-x_{2}\right|\left|x_{2}-x_{3}\right|\left|x_{3}-x_{4}\right|\left|x_{4}-x_{1}\right|\right)^{2 H^{\prime}-2}>0 .
\end{aligned}
$$

4. The noncentral limit theorem for the cubic variation of the Rosenblatt process. Denote by $L_{t}$ the kernel of the Rosenblatt process:

$$
L_{t}(x, y)=d(H) 1_{[0, t] \otimes^{\otimes 2}}(x, y) \int_{x \vee y}^{t} \partial_{1} K^{H^{\prime}}(s, x) \partial_{1} K^{H^{\prime}}(s, y) d s
$$

and recall the notation

$$
f_{i, N}(x, y)=L_{(i+1) / N}^{(H)}(x, y)-L_{i / N}^{(H)}(x, y) .
$$

We proved in the previous section that the dominant term of the statistics $V^{3, N}$ which gives its normalization is

$$
C(H)^{-1} N^{3 H-1} \sum_{i=0}^{N-1} I_{2}\left(g_{i, N}\right),
$$


where

$$
\begin{aligned}
g_{i, N} & =6\left\|f_{i, N}\right\|_{L^{2}\left([0,1]^{2}\right)}^{2} f_{i, N}+24\left(f_{i, N} \otimes_{1} f_{i, N}\right) \otimes_{1} f_{i, N} \\
& =3 N^{-2 H} f_{i, N}+24\left(f_{i, N} \otimes_{1} f_{i, N}\right) \otimes_{1} f_{i, N}=: 3 g_{i, N}^{(1)}+24 g_{i, N}^{(2)} .
\end{aligned}
$$

More precisely, it follows from the proof of Proposition 1 that

$$
\begin{aligned}
& \mathbf{E}\left[N^{1-H}\left(N^{3 H-1} \sum_{i=0}^{N-1} I_{2}\left(g_{i, N}^{(1)}\right)\right)\right]^{2}=N^{4 H} A_{1, N}^{(2)} \underset{N \rightarrow \infty}{\longrightarrow} \frac{1}{8} \\
& \mathbf{E}\left[N^{1-H}\left(N^{3 H-1} \sum_{i=0}^{N-1} I_{2}\left(g_{i, N}^{(2)}\right)\right)\right]^{2}=N^{4 H} A_{3, N}^{(2)} \underset{N \rightarrow \infty}{\longrightarrow} \frac{H^{2}(2 H-1)^{2}}{8}\left(C^{\prime}(H)\right)^{2} .
\end{aligned}
$$

Consequently, the limit of the sequence $V^{3, N}$ is the same as the limit of the sequence

$$
C(H)^{-1} N^{1-H} N^{3 H-1}\left(3 \sum_{i=0}^{N-1} I_{2}\left(g_{i, N}^{(1)}\right)+24 \sum_{i=0}^{N-1} I_{2}\left(g_{i, N}^{(2)}\right)\right)
$$

We prove here our main result.

Theorem 1. The renormalized cubic variation statistics based on the Rosenblatt process $N^{1-H} V^{3, N}$ with $V^{3, N}$ given by (10) converges in $L^{2}(\Omega)$ as $N \rightarrow \infty$ to the Rosenblatt random variable $D(H) Z_{1}^{(H)}$, where $D(H)=$ $C(H)^{-1}\left(3+24 d(H)^{2} a(H)^{2} C^{\prime}(H)\right)$.

P r o o f. To find the limit of $N^{1-H} V^{3, N}$ we need to study the convergence of $N^{1-H} N^{3 H-1} \sum_{i=0}^{N-1} I_{2}\left(g_{i, N}^{(1)}\right)$ and of $N^{1-H} N^{3 H-1} \sum_{i=0}^{N-1} I_{2}\left(g_{i, N}^{(2)}\right)$.

Is easy to treat the first part. In fact we have

$$
N^{1-H} N^{3 H-1} \sum_{i=0}^{N-1} I_{2}\left(g_{i, N}^{(1)}\right)=N^{2 H} \sum_{i=0}^{N-1} N^{-2 H} I_{2}\left(f_{i, N}\right)=\sum_{i=0}^{N-1} I_{2}\left(f_{i, N}\right)=Z_{1}^{H},
$$

where $Z_{1}^{H}$ is a Rosenblatt random variable with self-similarity order $H$.

We find then the limit of the second part of the dominant term. We have

$$
N^{1-H} N^{3 H-1} \sum_{i=0}^{N-1} I_{2}\left(g_{i, N}^{(2)}\right)=N^{2 H} \sum_{i=0}^{N-1} I_{2}\left(\left(f_{i, N} \otimes_{1} f_{i, N}\right) \otimes_{1} f_{i, N}\right) .
$$

Introduce the notation

$$
\begin{aligned}
l^{H^{\prime}}(x, y, z, t) & :=\partial_{1} K^{H^{\prime}}(x, y) \partial_{1} K^{H^{\prime}}(z, t), \\
l_{0}^{H^{\prime}}(x, y, z) & :=\partial_{1} K^{H^{\prime}}(x, y) \partial_{1} K^{H^{\prime}}(x, z)=l^{H^{\prime}}(x, y, x, z), \\
l_{1}^{H^{\prime}}(x, y, z) & :=\partial_{1} K^{H^{\prime}}(x, z) \partial_{1} K^{H^{\prime}}(y, z)=l^{H^{\prime}}(x, z, y, z) .
\end{aligned}
$$


Using relations (19) and (20) we get

$$
\begin{aligned}
&\left(\left(f_{i, N} \otimes_{1} f_{i, N}\right) \otimes_{1} f_{i, N}\right)\left(y_{1}, y_{2}\right)=d(H)^{3} a(H)^{2} 1_{[0, i / N]}^{\otimes 2}\left(y_{1}, y_{2}\right) \\
& \quad \times \int_{I_{i}^{3}} d u_{1} d u_{2} d u_{3} l^{H^{\prime}}\left(u_{1}, y_{1}, u_{3}, y_{2}\right)\left[\left|u_{1}-u_{2}\right|\left|u_{2}-u_{3}\right|\right]^{2 H^{\prime}-2} \\
&=: b_{i, N}^{(1)}\left(y_{1}, y_{2}\right)+b_{i, N}^{(2)}\left(y_{1}, y_{2}\right)
\end{aligned}
$$

with

$$
\begin{aligned}
b_{i, N}^{(1)}\left(y_{1}, y_{2}\right)= & d(H)^{3} a(H)^{2} 1_{[0, i / N]}^{\otimes 2}\left(y_{1}, y_{2}\right) \\
& \times \int_{I_{i}^{3}} d u_{1} d u_{2} d u_{3} l^{H^{\prime}}\left(u_{1}, y_{1}, u_{3}, y_{2}\right)\left[\left|u_{1}-u_{2}\right|\left|u_{2}-u_{3}\right|\right]^{2 H^{\prime}-2}
\end{aligned}
$$

and

$$
\begin{aligned}
& b_{i, N}^{(2)}\left(y_{1}, y_{2}\right)= d(H)^{3} a(H)^{2} \\
& \times\left[1_{I_{i}}\left(y_{1}\right) 1_{[0, i / N]}\left(y_{2}\right) \int_{y_{1}}^{(i+1) / N} d u_{1} \int_{I_{i}^{2}} d u_{2} d u_{3} l^{H^{\prime}}\left(u_{1}, y_{1}, u_{3}, y_{2}\right)\right. \\
& \times\left[\left|u_{1}-u_{2}\right|\left|u_{2}-u_{3}\right|\right]^{2 H^{\prime}-2} \\
&+ 1_{[0, i / N]}\left(y_{1}\right) 1_{I_{i}}\left(y_{2}\right) \int_{I_{i}^{2}} d u_{1} d u_{2} \int_{y_{2}}^{(i+1) / N} d u_{3} l^{H^{\prime}}\left(u_{1}, y_{1}, u_{3}, y_{2}\right) \\
& \times\left[\left|u_{1}-u_{2}\right|\left|u_{2}-u_{3}\right|\right]^{2 H^{\prime}-2} \\
&+1_{I_{i}^{2}}\left(y_{1}, y_{2}\right) \int_{y_{1}}^{(i+1) / N} d u_{1} \int_{I_{i}} d u_{2} \int_{y_{2}}^{(i+1) / N} d u_{3} l^{H^{\prime}}\left(u_{1}, y_{1}, u_{3}, y_{2}\right) \\
&
\end{aligned}
$$

We show that $I_{2}\left(N^{2 H} \sum_{i=0}^{N-1} b_{i, N}^{(2)}\right)$ converges to zero in $L^{2}(\Omega)$ and it makes no contribution to the limit. Indeed,

$$
\begin{aligned}
& \mathbf{E}\left(I_{2}\left(N^{2 H} \sum_{i=0}^{N-1} b_{i, N}^{(2)}\right)\right)^{2}=2 N^{4 H} \int_{0}^{1} \int_{0}^{1} d y_{1} d y_{2}\left(\sum_{i=0}^{N-1} b_{i, N}^{2}\left(y_{1}, y_{2}\right)\right)^{2} \\
& \leqslant 2 d(H)^{6} a(H)^{4} N^{4 H} \\
& \times\left(\sum_{i=0}^{N-1} \int_{0}^{1} \int_{0}^{1} d y_{1} d y_{2} \int_{I_{i}^{6}} d u_{1} d u_{1}^{\prime} d u_{2} d u_{2}^{\prime} d u_{3} d u_{3}^{\prime} 1_{\left[y_{1},(i+1) / N\right]^{2}}\left(u_{1}, u_{1}^{\prime}\right)\right. \\
& \times 1_{\left[y_{2},(i+1) / N\right]^{2}}\left(u_{3}, u_{3}^{\prime}\right) l_{1}^{H^{\prime}}\left(u_{1}, u_{1}^{\prime}, y_{1}\right) l_{1}^{H^{\prime}}\left(u_{3}, u_{3}^{\prime}, y_{2}\right) \\
&\left.\times\left[\left|u_{1}-u_{2} \| u_{2}-u_{3}\right|\left|u_{1}^{\prime}-u_{2}^{\prime}\right|\left|u_{2}^{\prime}-u_{3}^{\prime}\right|\right]^{2 H^{\prime}-2}\right) \\
& \leqslant 2 d(H)^{6} a(H)^{4} N^{4 H}\left(\sum_{i=0}^{N-1} \int_{I_{i}^{6}} d u_{1} d u_{1}^{\prime} d u_{2} d u_{2}^{\prime} d u_{3} d u_{3}^{\prime}\right.
\end{aligned}
$$




$$
\begin{gathered}
\times\left[\left|u_{1}-u_{2}\right|\left|u_{2}-u_{3}\right|\left|u_{1}^{\prime}-u_{2}^{\prime} \| u_{2}^{\prime}-u_{3}^{\prime}\right|\right]^{2 H^{\prime}-2} \\
\left.\times \int_{0}^{u_{1} \wedge u_{1}^{\prime}} d y_{1} l_{1}^{H^{\prime}}\left(u_{1}, u_{1}^{\prime}, y_{1}\right) \int_{0}^{u_{3} \wedge u_{3}^{\prime}} d y_{2} l_{1}^{H^{\prime}}\left(u_{3}, u_{3}^{\prime}, y_{2}\right)\right) \\
\leqslant 2 d(H)^{6} a(H)^{4} N^{4 H}\left(\sum_{i=0}^{N-1} \int_{I_{i}^{6}} d u_{1} d u_{1}^{\prime} d u_{2} d u_{2}^{\prime} d u_{3} d u_{3}^{\prime}\right. \\
\left.\times\left[\left|u_{1}-u_{2}\left\|u_{2}-u_{3}\right\| u_{1}^{\prime}-u_{2}^{\prime}\right|\left|u_{2}^{\prime}-u_{3}^{\prime}\right|\left|u_{1}-u_{1}^{\prime} \| u_{1}-u_{3}^{\prime}\right|\right]^{2 H^{\prime}-2}\right) \\
\leqslant 2 d(H)^{6} a(H)^{4} N^{4 H} N N^{-6} N^{12-12 H^{\prime}}\left(\int_{[0,1]^{6}} d v_{1} d v_{1}^{\prime} d v_{2} d v_{2}^{\prime} d v_{3} d v_{3}^{\prime}\right. \\
\left.\times\left[\left|v_{1}-v_{2}\right|\left|v_{2}-v_{3}\right|\left|v_{1}^{\prime}-v_{2}^{\prime}\right|\left|v_{2}^{\prime}-v_{3}^{\prime}\right|\left|v_{1}-v_{1}^{\prime}\right|\left|v_{1}-v_{3}^{\prime}\right|\right]^{2 H^{\prime}-2}\right) \\
\leqslant 2 c d(H)^{6} a(H)^{4} N^{1-2 H} .
\end{gathered}
$$

Combining it with the fact that $H>1 / 2$, we conclude that

$$
I_{2}\left(N^{2 H} \sum_{i=0}^{N-1} b_{i, N}^{(2)}\right) \underset{N \rightarrow \infty}{\longrightarrow} 0 \text { in } L^{2}(\Omega),
$$

and then we need to find the limit of

$$
\begin{aligned}
N^{2 H} \sum_{i=0}^{N-1} b_{i, N}^{(1)}= & d(H)^{3} a(H)^{2} N^{2 H} \sum_{i=0}^{N-1} 1_{[0, i / N]^{2}}\left(y_{1}, y_{2}\right) \\
& \times \int_{I_{i}^{3}} d u_{1} d u_{2} d u_{3} l^{H^{\prime}}\left(u_{1}, y_{1}, u_{3}, y_{2}\right)\left[\left|u_{1}-u_{2}\right|\left|u_{2}-u_{3}\right|\right]^{2 H^{\prime}-2} \\
= & d(H)^{3} a(H)^{2} \sum_{i=0}^{N-1} 1_{[0, i / N]^{2}}\left(y_{1}, y_{2}\right) N^{-1} \\
& \times \int_{[0,1]^{3}} d v_{1} d v_{2} d v_{3} l^{H^{\prime}}\left(\frac{v_{1}+i}{N}, y_{1}, \frac{v_{3}+i}{N}, y_{2}\right) \\
& \times\left[\left|v_{1}-v_{2} \| v_{2}-v_{3}\right|\right]^{2 H^{\prime}-2} .
\end{aligned}
$$

The last sequence has the same pointwise limit (for every $y_{1}, y_{2}$ ) as

$$
\begin{gathered}
d(H)^{3} a(H)^{2} \int_{[0,1]^{3}} d v_{1} d v_{2} d v_{3}\left[\left|v_{1}-v_{2}\right|\left|v_{2}-v_{3}\right|\right]^{2 H^{\prime}-2} \\
\times \sum_{i=0}^{N-1} 1_{[0, i / N]^{2}}\left(y_{1}, y_{2}\right) N^{-1} l^{H^{\prime}}\left(\frac{i}{N}, y_{1}, \frac{i}{N}, y_{2}\right) .
\end{gathered}
$$

This last term is a Riemann sum that converges to

$$
\begin{aligned}
& d(H)^{3} a(H)^{2} \int_{[0,1]^{3}} d v_{1} d v_{2} d v_{3}\left[\left|v_{1}-v_{2}\right|\left|v_{2}-v_{3}\right|\right]^{2 H^{\prime}-2} \\
& \quad \times \int_{y_{1} \vee y_{2}}^{1} d x l^{H^{\prime}}\left(x, y_{1}, x, y_{2}\right)
\end{aligned}
$$




$$
\begin{aligned}
& =d(H)^{3} a(H)^{2} C^{\prime}(H) \int_{y_{1} \vee y_{2}}^{1} d x \partial_{1} K^{H^{\prime}}\left(x, y_{1}\right) \partial_{1} K^{H^{\prime}}\left(x, y_{2}\right) \\
& =d(H)^{2} a(H)^{2} C^{\prime}(H) L_{1}^{(H)}\left(y_{1}, y_{2}\right),
\end{aligned}
$$

where $L_{1}^{(H)}$ is the standard kernel of the Rosenblatt process, see (6).

We need a Cauchy sequence argument as in [20] to conclude the proof. That is, we will show that the sequence $N^{2 H} \sum_{i=0}^{N-1} b_{i, N}^{(1)}$ (or equivalently $\left.N^{2 H} \sum_{i=0}^{N-1} g_{i, N}^{(2)}\right)$ is Cauchy in the Hilbert space $L^{2}\left([0,1]^{2}\right)$. This will imply that the sequence of random variables $I_{2}\left(N^{2 H} \sum_{i=0}^{N-1} g_{i, N}^{(2)}\right)$ is Cauchy, so convergent, in the space $L^{2}(\Omega)$ and it is easy to deduce that its limit coincides with the multiple integral of the pointwise limit of the kernel. We compute, for $M, N \geqslant 1$

$$
\begin{aligned}
& \left\|N^{2 H} \sum_{i=0}^{N-1} b_{i, N}^{(1)}-M^{2 H} \sum_{i=0}^{M-1} b_{i, M}^{(1)}\right\|_{L^{2}\left([0,1]^{2}\right)}^{2} \\
& =d(H)^{6} a(H)^{4}\left[N^{4 H} \sum_{i, j=0}^{N-1} \int_{I_{i}^{3}} \int_{I_{j}^{3}} d u_{1} d u_{2} d u_{3} d u_{1}^{\prime} d u_{2}^{\prime} d u_{3}^{\prime}\right. \\
& \times\left[\left|u_{1}-u_{2}\right|\left|u_{2}-u_{3}\right|\left|u_{1}^{\prime}-u_{2}^{\prime}\right|\left|u_{2}^{\prime}-u_{3}^{\prime}\right|\right]^{2 H^{\prime}-2} \\
& \times \int_{0}^{u_{1} \wedge u_{1}^{\prime}} d y_{1} \int_{0}^{u_{3} \wedge u_{3}^{\prime}} d y_{2} l^{H^{\prime}}\left(u_{1}, y_{1}, u_{3}, y_{2}\right) l^{H^{\prime}}\left(u_{1}^{\prime}, y_{1}, u_{3}^{\prime}, y_{2}\right) \\
& +M^{4 H} \sum_{i, j=0}^{M-1} \int_{I_{i}^{3}} \int_{I_{j}^{3}} d u_{1} d u_{2} d u_{3} d u_{1}^{\prime} d u_{2}^{\prime} d u_{3}^{\prime} \\
& \times\left[\left|u_{1}-u_{2}\right|\left|u_{2}-u_{3}\right|\left|u_{1}^{\prime}-u_{2}^{\prime}\right|\left|u_{2}^{\prime}-u_{3}^{\prime}\right|\right]^{2 H^{\prime}-2} \\
& \times \int_{0}^{u_{1} \wedge u_{1}^{\prime}} d y_{1} \int_{0}^{u_{3} \wedge u_{3}^{\prime}} d y_{2} l^{H^{\prime}}\left(u_{1}, y_{1}, u_{3}, y_{2}\right) l^{H^{\prime}}\left(u_{1}^{\prime}, y_{1}, u_{3}^{\prime}, y_{2}\right) \\
& -2 N^{2 H} M^{2 H} \sum_{i=0}^{N-1} \sum_{j=0}^{M-1} \int_{I_{i}^{3}} \int_{I_{j}^{3}} d u_{1} d u_{2} d u_{3} d u_{1}^{\prime} d u_{2}^{\prime} d u_{3}^{\prime} \\
& \times\left[\left|u_{1}-u_{2}\right|\left|u_{2}-u_{3}\right|\left|u_{1}^{\prime}-u_{2}^{\prime}\right|\left|u_{2}^{\prime}-u_{3}^{\prime}\right|\right]^{2 H^{\prime}-2} \\
& \left.\times \int_{0}^{u_{1} \wedge u_{1}^{\prime}} d y_{1} \int_{0}^{u_{3} \wedge u_{3}^{\prime}} d y_{2} l^{H^{\prime}}\left(u_{1}, y_{1}, u_{3}, y_{2}\right) l^{H^{\prime}}\left(u_{1}^{\prime}, y_{1}, u_{3}^{\prime}, y_{2}\right)\right] \\
& =d(H)^{6} a(H)^{4}\left[N^{4 H} \sum_{i, j=0}^{N-1} \int_{I_{i}^{3}} \int_{I_{j}^{3}} d u_{1} d u_{2} d u_{3} d u_{1}^{\prime} d u_{2}^{\prime} d u_{3}^{\prime}\right. \\
& \times\left[\left|u_{1}-u_{2}\right|\left|u_{2}-u_{3}\right|\left|u_{1}^{\prime}-u_{2}^{\prime}\right|\left|u_{2}^{\prime}-u_{3}^{\prime}\right|\left|u_{1}-u_{1}^{\prime}\right|\left|u_{3}-u_{3}^{\prime}\right|\right]^{2 H^{\prime}-2} \\
& +M^{4 H} \sum_{i, j=0}^{M-1} \int_{I_{i}^{3}} \int_{I_{j}^{3}} d u_{1} d u_{2} d u_{3} d u_{1}^{\prime} d u_{2}^{\prime} d u_{3}^{\prime} \\
& \times\left[\left|u_{1}-u_{2}\right|\left|u_{2}-u_{3}\right|\left|u_{1}^{\prime}-u_{2}^{\prime}\right|\left|u_{2}^{\prime}-u_{3}^{\prime}\right|\left|u_{1}-u_{1}^{\prime} \| u_{3}-u_{3}^{\prime}\right|\right]^{2 H^{\prime}-2}
\end{aligned}
$$




$$
\begin{aligned}
& -2 N^{2 H} M^{2 H} \sum_{i=0}^{N-1} \sum_{j=0}^{M-1} \int_{I_{i}^{3}} \int_{I_{j}^{3}} d u_{1} d u_{2} d u_{3} d u_{1}^{\prime} d u_{2}^{\prime} d u_{3}^{\prime} \\
& \left.\times\left[\left|u_{1}-u_{2}\right|\left|u_{2}-u_{3}\right|\left|u_{1}^{\prime}-u_{2}^{\prime}\right|\left|u_{2}^{\prime}-u_{3}^{\prime}\right|\left|u_{1}-u_{1}^{\prime}\right|\left|u_{3}-u_{3}^{\prime}\right|\right]^{2 H^{\prime}-2}\right]
\end{aligned}
$$

and this is equal to

$$
\begin{aligned}
& d(H)^{6} a(H)^{4}\left[N^{-2 H} \sum_{i, j=0}^{N-1} \int_{[0,1]^{6}} d u_{1} d u_{2} d u_{3} d u_{1}^{\prime} d u_{2}^{\prime} d u_{3}^{\prime}\right. \\
& \times\left[\left|v_{1}-v_{2}\right|\left|v_{2}-v_{3}\right|\left|v_{1}^{\prime}-v_{2}^{\prime}\right|\left|v_{2}^{\prime}-v_{3}^{\prime}\right|\left|v_{1}-v_{1}^{\prime}+i-j \| v_{3}-v_{3}^{\prime}+i-j\right|\right]^{2 H^{\prime}-2} \\
& +M^{-2 H} \sum_{i, j=0}^{M-1} \int_{[0,1]^{6}} d u_{1} d u_{2} d u_{3} d u_{1}^{\prime} d u_{2}^{\prime} d u_{3}^{\prime} . \\
& \times\left[\left|v_{1}-v_{2}\right|\left|v_{2}-v_{3}\right|\left|v_{1}^{\prime}-v_{2}^{\prime}\right|\left|v_{2}^{\prime}-v_{3}^{\prime}\right|\left|v_{1}-v_{1}^{\prime}+i-j \| v_{3}-v_{3}^{\prime}+i-j\right|\right]^{2 H^{\prime}-2} \\
& -2 N^{-1} M^{-1} \sum_{i=0}^{N-1} \sum_{j=0}^{M-1} \int_{[0,1]^{6}} d u_{1} d u_{2} d u_{3} d u_{1}^{\prime} d u_{2}^{\prime} d u_{3}^{\prime} \\
& \times\left[\left|v_{1}-v_{2}\right|\left|v_{2}-v_{3}\right|\left|v_{1}^{\prime}-v_{2}^{\prime}\right|\left|v_{2}^{\prime}-v_{3}^{\prime}\right|\left|\frac{v_{1}}{N}-\frac{v_{1}^{\prime}}{M}+\frac{i}{N}-\frac{j}{M}\right|\right. \\
& \left.\left.\quad \times\left|\frac{v_{3}}{N}-\frac{v_{3}^{\prime}}{M}+\frac{i}{N}-\frac{j}{M}\right|\right]^{2 H^{\prime}-2}\right] .
\end{aligned}
$$

In the same way as above this last term as $N \rightarrow \infty$ and $M \rightarrow \infty$ converges to

$$
\begin{aligned}
& d(H)^{6} a(H)^{4}\left[2 \int_{0}^{1}(1-x) x^{2 H-2} d x+2 \int_{0}^{1}(1-y) y^{2 H-2} d y\right. \\
& \left.\quad+2 \int_{0}^{1} \int_{0}^{1}|x-y|^{2 H-2} d x d y\right] \\
& =\frac{1}{H(2 H-1)}+\frac{1}{H(2 H-1)}-\frac{2}{H(2 H-1)}=0
\end{aligned}
$$

We obtained that $\left\{N^{2 H} \sum_{i=0}^{N-1} b_{i, N}^{(1)}, N \geqslant 0\right\}$ is a Cauchy sequence. Theorem 1 is proved.

\section{СПИСОК ЛИТЕРАТУРЫ}

1. Beran J. Statistics for Long-Memory Processes. New York: Chapman \& Hall, 1994, $315 \mathrm{p}$.

2. Breton J.-C., Nourdin I. Error bounds on the non-normal approximation of Hermite power variations of fractional Brownian motion. - Electron. Commun. Probab., 2008, v. 13, p. $482-493$.

3. Breuer P., Major P. Central limit theorems for nonlinear functionals of Gaussian fields. - J. Multivariate Anal., 1983, v. 13, № 3, p. 425-441. 
4. Chronopoulou A., Tudor C.A., Viens F. Self-similarity parameter estimation and reproduction property for non-Gaussian Hermite processes. arXiv: 0807.1208.

5. Chronopoulou A., Viens F., Tudor C. A. Variations and Hurst index estimation for a Rosenblatt process using longer filters. - Electron. J. Statist., 2009, v. 3, p. 13931435 .

6. Coeurjolly J.-F. Estimating the parameters of a fractional Brownian motion by discrete variations of its sample paths. - Statist. Inference Stoch. Process., 2001, v. 4, № 2, p. 199-227.

7. Dobrushin R. L., Major P. Non-central limit theorems for non-linear functionals of Gaussian fields. - Z. Wahrscheinlichkeitstheor. verw. Geb., 1979, v. 50, № 1, p. 27-52.

8. Embrechts P., Maejima M. Selfsimilar Processes. Princeton: Princeton Univ. Press, 2002, $111 \mathrm{p}$

9. Jacod J., Shiryaev A.N. Limit Theorems for Stochastic Processes. 2nd ed. Berlin: Springer-Verlag, 2003, 661 p. (Grundlehren Math. Wiss., v. 288.)

10. Giraitis L., Surgailis D. CLT and other limit theorems for functionals of Gaussian processes. - Z. Wahrschienlichkeitstheor. verw. Geb., 1985, v. 70, № 2, p. 191-212.

11. Maejima M., Tudor C.A. Wiener integrals with respect to the Hermite process and a non-central limit theorem. - Stochastic Anal. Appl., 2007, v. 25, № 5, p. 1043-1056.

12. Nourdin I., Nualart D., Tudor C.A Central and non-central limit theorems for weighted power variations of the fractional Brownian motion. arXiv: 0710.5639.

13. Nourdin I., Peccati G., Réveillac A. Multivariate normal approximation using Stein's method and Malliavin calculus. - Ann. Inst. H. Poincaré, 2010, v. 46, № 1, p. 45-58.

14. Nualart D. Malliavin Calculus and Related Topics. Berlin: Springer-Verlag, 2006, $382 \mathrm{p}$.

15. Nualart D., Ortiz-Latorre S. Central limit theorems for multiple stochastic integrals and Malliavin calculus. - Stochastic Process. Appl., 2008, v. 118, № 4, p. 614-628.

16. Prokhorov Yu. V., Statulevicius V. (Eds.). Limit Theorems in Probability Theory. Berlin: Springer, 2006.

17. Samorodnitsky G., Taqqu M.S. Stable Non-Gaussian Random Processes. New York: Chapman \& Hall, 1994, $632 \mathrm{p}$.

18. Taqqu M.S. Weak convergence to fractional Brownian motion and to the Rosenblatt process. - Z. Wahrscheinlichkeitstheor. verw. Geb., 1975, v. 31, p. 287-302.

19. Tudor C. A. Analysis of the Rosenblatt process. - ESAIM Probab. Statist., 2008, v. 12 , p. $230-257$.

20. Tudor C. A., Viens F. G. Variations and estimators for self-similarity parameters via Malliavin calculus. - Ann. Probab., 2009, v. 37, №6, p. 2093-2134.

Поступила в редакцию

27.XI.2008

Исправленный вариант

9.XI.2009 